\title{
Solar abundances of oxygen and neon derived from solar wind observations
}

\begin{abstract}
P. Bochsler
Physikalisches Institut, Universität Bern, Sidlerstrasse 5, 3012 Bern, Switzerland

e-mail: bochsler@soho.unibe.ch

Received 2 May 2007 / Accepted 2 June 2007

ABSTRACT

Context. Recently, a revision of the solar abundances of $\mathrm{C}, \mathrm{N}$, and $\mathrm{O}$ to substantially lower values has led to a controversy on solar opacities in the solar standard model and to the suggestion to revise the solar abundance of neon upward by as much as a factor of 1.6 leading to enhanced solar neon/oxygen abundance ratios by a factor of 3. Neon and oxygen are neighboring elements with easily defined charged states in the solar wind, and they have been well identified and measured for over two decades in the solar wind under many circumstances and with several instruments. The solar wind $\mathrm{Ne} / \mathrm{O}$ ratio is 0.14 with a conservative error estimate of \pm 0.03 , consistent with the coronal value derived from solar energetic particle measurements.

Aims. We investigate, whether solar wind observations are consistent with the newly proposed elemental solar abundances of neon and oxygen.

Methods. The solar helium abundance has been derived from helioseismological observations. Helium and neon abundances in the solar wind have been well determined with the Apollo Foil experiments and, more recently, confirmed with the Genesis sample return mission. With these observations and the neon/oxygen solar wind abundance ratio determined by in-situ mass-spectrometry and using a simple theoretical model of Coulomb-drag fractionation for the solar wind, we estimate solar abundances for neon and oxygen. Results. From the variability of the helium/oxygen and the helium/neon ratio in the solar wind and from theoretical considerations, we conclude that the helium/neon ratio in the outer solar convective zone is $900 \pm 110$. Our best estimates of the solar neon and oxygen abundances in logarithmic dex-units are $[\mathrm{Ne}]=7.96 \pm 0.13$, and $[\mathrm{O}]=8.87 \pm 0.11$.

Conclusions. Our solar neon/oxygen abundance ratio is consistent with the ratio derived from EUV-spectra from SOHO/CDS. However, our absolute abundance for oxygen is only marginally compatible with the newly derived value for oxygen, and our neon value is clearly incompatible with the recently proposed enhancement of the solar neon abundance.
\end{abstract}

Key words. Sun: solar wind - Sun: photosphere - Sun: corona - Sun: abundances

\section{Introduction}

Recently, Asplund et al. (2005) have proposed a new list of solar abundances, in which the contributions of heavy elements have been significantly reduced. As a consequence of adopting these new abundances for calculating solar opacities, one finds that these opacities are no longer compatible with the ones derived from helioseismological observations (Bahcall et al. 2005; Antia \& Basu 2006). In another recent article, Bochsler et al. (2006) have argued that solar abundances inferred from solar wind measurements for oxygen and neon are also incompatible with the new values given by Asplund et al. (2005). In this paper we provide further evidence and make an attempt to substantiate the claims made by Bochsler et al. (2006), adding a detailed analysis of uncertainties in the solar-wind-inferred values. The principal ingredients for this study are the helioseismologically determined solar helium abundance, the solar wind isotopic abundance ratios ${ }^{3} \mathrm{He} /{ }^{4} \mathrm{He}$ and ${ }^{20} \mathrm{Ne} /{ }^{22} \mathrm{Ne}$, which have well been determined by means of the Apollo Foil experiments (cf. Geiss et al. 2004), as well as the elemental abundance ratios $\mathrm{He} / \mathrm{Ne}$ and $\mathrm{Ne} / \mathrm{O}$, which have been determined with the Apollo foils and the ISEE-3 Ion Composition Instrument (Kunz 1983; Bochsler et al. 1986) and several other in-situ experiments, respectively. Hence, for our determination we use measurements, which do not depend on photospheric observations, nor on atomic properties, such as ft-values, nor on photospheric models. However, we will have to make use of a model of elemental and isotopic fractionation in the solar wind, i.e., the model of Bodmer \& Bochsler (1998).

The abundance of helium in the outer convective zone (OCZ) of the Sun is not in question, and the helium mass fraction $Y=$ $0.245 \pm 0.005$ (Gough 2006) has an uncertainty which is an order of magnitude below the uncertainties of all other solar elemental abundances. Helium is also routinely well determined by in-situ mass spectrometry in the solar wind (e.g. Kasper et al. 2006), and it has been known since the first composition measurements that helium is underabundant relative to the source in the OCZ by a factor of 2 on average.

Helium is special in two respects in comparison to all other abundant species in the solar wind: 1) It has the highest first ionization potential (FIP) of all elements; and 2) ${ }^{4} \mathrm{He}^{++}$has the least favourable Coulomb-drag factor of all abundant species in typical solar wind flows. In principle, both properties could negatively affect the representation of helium in the solar wind. To infer solar abundances of other elements, e.g., $\mathrm{O}$ or $\mathrm{Ne}$, from solar wind observations one has to assess possible fractionation mechanisms, which might affect elemental abundance ratios in the solar wind. Both the $\mathrm{He} / \mathrm{O}$-abundance ratio as well as the $\mathrm{He} / \mathrm{Ne}$-ratio have been determined in many instances, and one can safely give average ratios with uncertainties of the order of $20 \%$. The most reliable value for the helium/neon ratio in the solar wind dates back to the work of Geiss et al. (1972), who found 


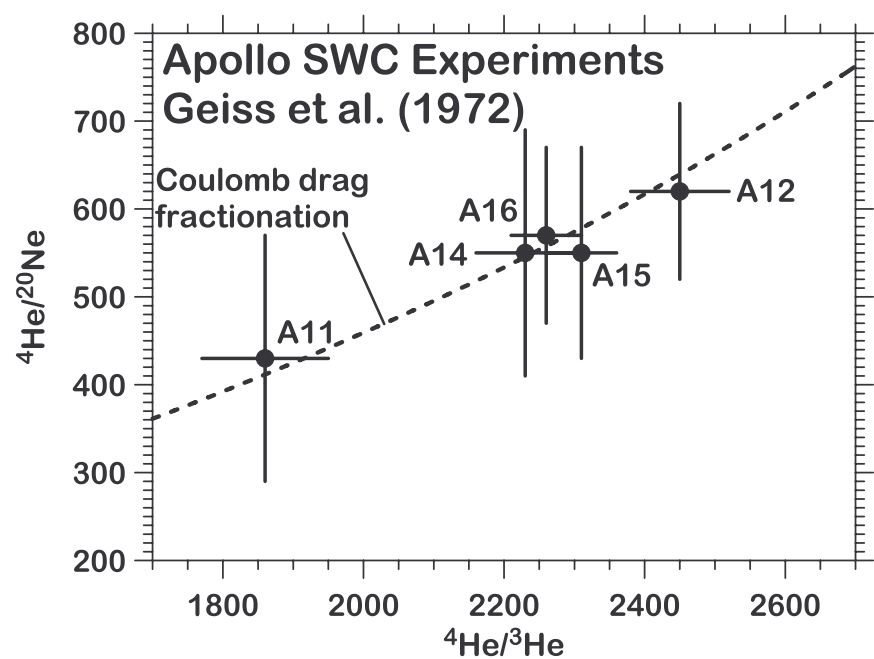

Fig. 1. Abundance ratios in the solar wind, obtained from the Apollo foil experiment (Geiss et al. 1972). The correlated variability indicates that the fractionation mechanism operates on both the elemental and the isotopic ratio simultaneously.

Table 1. Some properties of solar-wind species.

\begin{tabular}{lccc}
\hline $\begin{array}{l}\text { Element } \\
\text { or } \\
\text { isotope }\end{array}$ & $\begin{array}{r}\text { FIP } \\
{[\mathrm{eV}]}\end{array}$ & $\begin{array}{c}\text { Most abundant } \\
\text { species in the } \\
\text { solar wind }\end{array}$ & $\begin{array}{c}\text { Coulomb } \\
\text { drag factor } \\
\mathrm{H}^{-1}\end{array}$ \\
\hline $\mathrm{H}$ & 13.6 & $\mathrm{H}^{+}$ & - \\
${ }^{3} \mathrm{He}$ & 24.6 & ${ }^{3} \mathrm{He}^{2+}$ & 1.155 \\
${ }^{4} \mathrm{He}$ & 24.6 & ${ }^{4} \mathrm{He}^{2+}$ & 0.720 \\
${ }^{16} \mathrm{O}$ & 13.6 & ${ }^{16} \mathrm{O}^{6+}$ & 1.397 \\
${ }^{20} \mathrm{Ne}$ & 21.6 & ${ }^{20} \mathrm{Ne}^{8+}$ & 2.016 \\
${ }^{22} \mathrm{Ne}$ & 21.6 & ${ }^{22} \mathrm{Ne}^{8+}$ & 1.788 \\
\hline
\end{tabular}

a ${ }^{4} \mathrm{He} /{ }^{20} \mathrm{Ne}$-ratio of $570 \pm 70$ (see also Geiss et al. 2004, and see Fig. 1). Preliminary results from the Genesis mission (see Grimberg et al. 2007) largely confirm the Apollo results. From the isotopic abundance measurements one derives an elemental ratio $\mathrm{He} / \mathrm{Ne}=530 \pm 65$. In order to infer the fractionation factor of neon relative to helium in the solar wind, one should have knowledge of the momentary helium/hydrogen ratio in the solar wind during the exposures. Unfortunately, no helium/hydrogen abundance ratio has been measured during the periods of the exposures of the Apollo foils. But from the compilation of averages by Feldman et al. (1978) for the corresponding periods we deduce a He/H-ratio of $0.040 \pm 0.012$. From this we infer that solar wind helium was depleted by a factor of 2.0 during the exposures. With the extreme assumption that neon was depleted by a similar factor from its solar abundance during the exposures, we find that with a $\mathrm{Ne} / \mathrm{H}$-value in the solar wind of $7.5 \times 10^{-5}$, the solar abundance could amount to as much as $[\mathrm{Ne}]=8.19$ in logarithmic dex units. On the other hand, assuming the extreme alternative, i.e., that due to its particularly unfavourable properties only helium, but not neon, is depleted in the solar wind, would mean that the $\mathrm{Ne} / \mathrm{H}$-value in the solar wind is representative for the source, i.e., $[\mathrm{Ne}]=7.89$. Evidently, reality must lie somewhere in between. In the following, we will try to constrain the ranges of uncertainty for the solar neon and oxygen abundances.

\section{Coulomb drag versus FIP-effect}

Table 1 (from Bochsler et al. 2006) lists some properties of several species relevant for the following discussion. As before,
"FIP" stands for first ionization potential. The inverse Coulombdrag factor $\mathrm{H}$ is defined with Eq. (1), with $\mathrm{A}$ and $\mathrm{Q}$ denoting atomic weight and charge state of the species in elementary units:

$H=: \frac{2 A-Q-1}{Q^{2}} \sqrt{\frac{A+1}{A}}$.

Note that all species heavier than hydrogen listed in Table 1 are high-FIP elements.

To assess relevant fractionation mechanisms, we restrict the following discussion to two classes of processes:

- FIP-fractionation occurring in the chromosphere, where high-FIP elements are ionized first, before being transferred into the corona;

- (inefficient) Coulomb drag from protons as a counter agent to gravity in the lower corona, where heavy species on their way into the solar wind are no longer strongly coupled to field particles. Substantial observational evidence indicates that this mechanism operates in the context of variably diverging open magnetic flux tubes (see Kasper et al. 2007, for a more extensive discussion).

In order to disentangle the effects of the two mechanisms, which we consider candidates for elemental fractionation in the solar wind, we investigate the variability of elemental ratios in the solar wind under the influence of different parameters.

Some further explanations are in order:

We locate the effect of inefficient Coulomb drag as an agent for fractionation at a level in the corona, where collisions of protons (and electrons) with heavy ions become comparable to the solar wind expansion time and the characteristic ionization times for the relevant charge states of neon and oxygen. This justifies the choice of $\mathrm{O}^{6+}$ and $\mathrm{Ne}^{8+}$ in Table 1 as representative charge states; both helium-like configurations. Figure 2 illustrates the charge state evolution of the three elements, $\mathrm{He}, \mathrm{O}$, and $\mathrm{Ne}$ in a simple, radially expanding model of the inner corona. From $0.1 R_{\odot}$ upwards, charge state distributions begin to freeze, and, simultaneously, Coulomb drag becomes inefficient; both processes are steered by the magnetic flux expansion factor. The expansion of flux tubes in the low corona might contribute to the depletion of high-FIP elements and - at the same time - rule the equilibration of charge states. Thus the often observed correlation between the strength of the FIP-effect and the freeze-in temperatures could have a simple explanation.

Of the two mechanisms responsible for the depletion of helium in the solar wind - and to some smaller extent also of oxygen and neon - we clearly favour inefficient Coulomb drag. We do not attribute the helium depletion to an atomic effect, and see no reason to invoke a second high-FIP plateau for helium. There are several reasons for this conclusion:

As is obvious from Fig. 1, the helium/neon abundance variation observed with the Apollo foils is correlated with the isotopic composition of helium. Since isotope ratios vary most likely due to the variability of Coulomb drag, we attribute also the concomitant variability of the $\mathrm{He} / \mathrm{Ne}$-ratio to variable Coulomb drag. In fact, the observed slope in the correlation follows our simple Coulomb-drag model (dashed line) very well.

Oxygen and hydrogen have very similar first ionization potentials, and resonant charge exchange plays an important role in ionizing these species in a chromospheric gas (Geiss \& Bochsler 1985). Consequently, one would not expect 


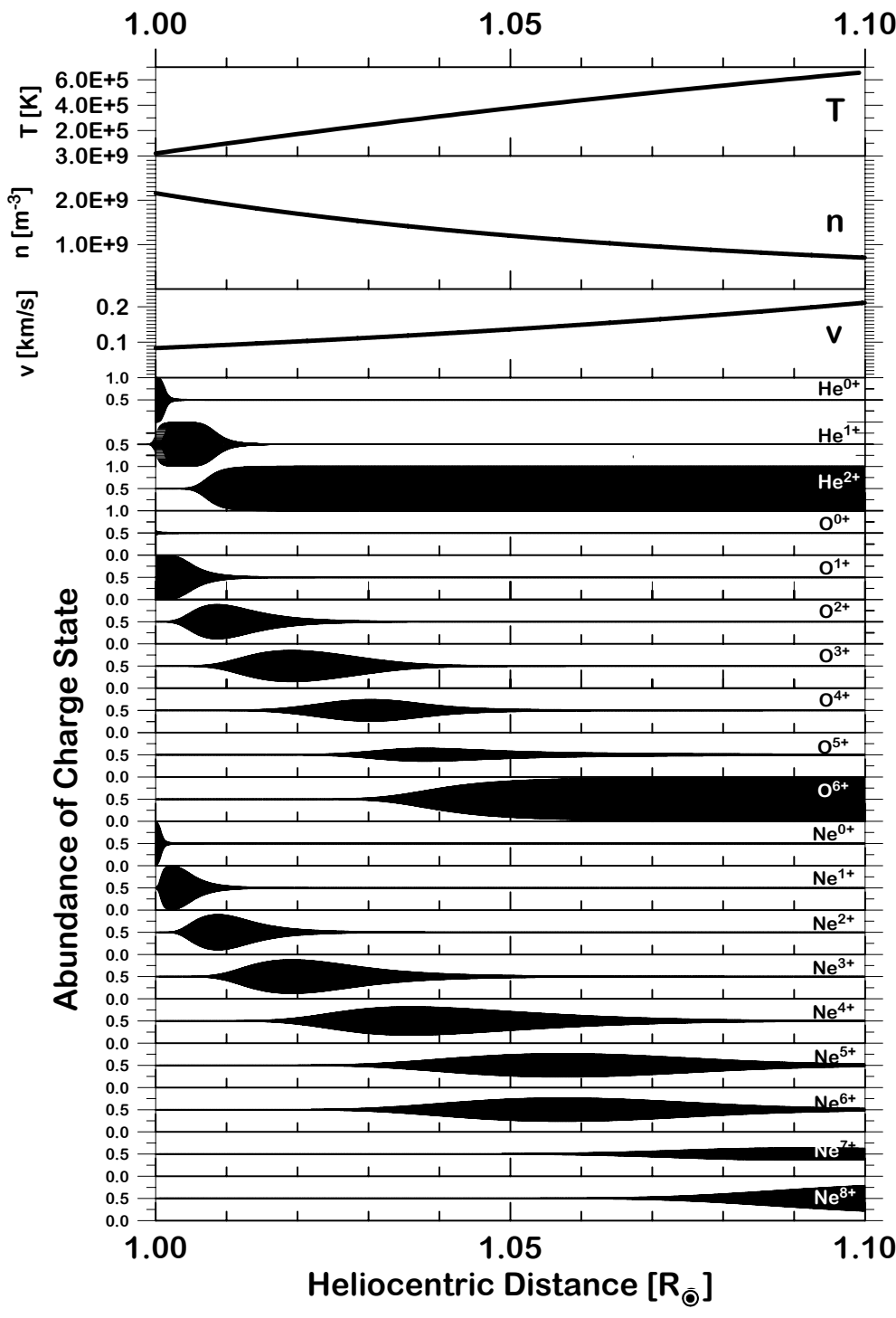

Fig. 2. Evolution of charge state distributions of helium, oxygen, and neon in the inner corona for a simple coronal expansion model. At $70000 \mathrm{~km}$ from the solar surface, the dominant charge states are $\mathrm{He}^{++}$, and the helium-like configurations $\mathrm{O}^{6+}$, and $\mathrm{Ne}^{8+}$. At this altitude characteristic Coulomb collision times of these species and the characteristic ionization times also become comparable to the solar wind expansion time scale.
FIP or the first ionization time to be involved in separating oxygen from hydrogen. Nevertheless, the oxygen/hydrogen ratio in the solar wind varies strongly, and the variation is strongly correlated with the helium/hydrogen ratio as is illustrated in Fig. 3 (Bochsler et al. 2006). If the oxygen/hydrogen variability is unlikely to be attributed to FIP-fractionation, it seems also unlikely that the helium/hydrogen variability is driven by FIPfractionation. Consequently, it seems also unlikely that any other variability of abundances among high-FIP elements is driven by FIP-fractionation.

The photoionization threshold of neon is not far below the ionization threshold of helium. Although the photoionization cross section of neon is generally larger than the one of helium, the energy dependencies are similar, and one would need a rather peculiar EUV-spectrum to cause variable discrimination between the two species (Bochsler et al. 2006).

\section{Extrapolation of solar oxygen and neon abundances from solar wind measurements}

Bodmer \& Bochsler (1998) estimate the depletion relative to protons of a species $i$ due to inefficient Coulomb drag (their Eq. (6) in a somewhat modified notation):

$\frac{f_{i}}{f_{p}}=1-H_{i} \cdot C$, where $f_{i}$ is the so-called fractionation factor of the species $i$, denoting the ratio of the flux of $i$ to the number density of $i$ in the source region, i.e., in the solar atmosphere. $H_{i}$ is the inverse Coulomb-drag coefficient given in Eq. (1), and $C$ is a function which depends on the instantaneous proton flux in the corona and a multiplicative friction coefficient, which is the same for all minor species. Dividing Eq. (2) by a similar equation for the species $j$, we can derive an equation for the fractionation ratio among two minor species $f_{i} / f_{j}$

$\frac{f_{i}}{f_{j}}=\frac{1-H_{i} \cdot C}{1-H_{j} \cdot C}$

The unknown quantity $C$ can be retrieved from Eq. (2) if, for instance, the fractionation factor for ${ }^{4} \mathrm{He}^{++}, f_{4}$, is known from observations. Hence, we can - as an example - compute

$\frac{f^{4} \mathrm{He}}{f^{20} \mathrm{Ne}}=\frac{f_{4} \cdot H_{4}}{\left(H_{4}-H_{20}\right)+f_{4} \cdot H_{20}}$.

The dashed curve in Fig. 1 delineates a similar relation between the variables $\frac{f_{4 \mathrm{He}}}{f_{20} 0_{\mathrm{Ne}}}$ and $\frac{f_{4_{\mathrm{He}}}}{f_{3_{\mathrm{He}}}}$. We have estimated an average $\mathrm{He} / \mathrm{H}$ fractionation factor during the exposures of the Apollo foil experiments $f_{4}=0.50 \pm 0.15$, assuming that solar wind $[\mathrm{He} / \mathrm{H}]$ was on average close to 0.04 . Using Eq. (4) and the values of 


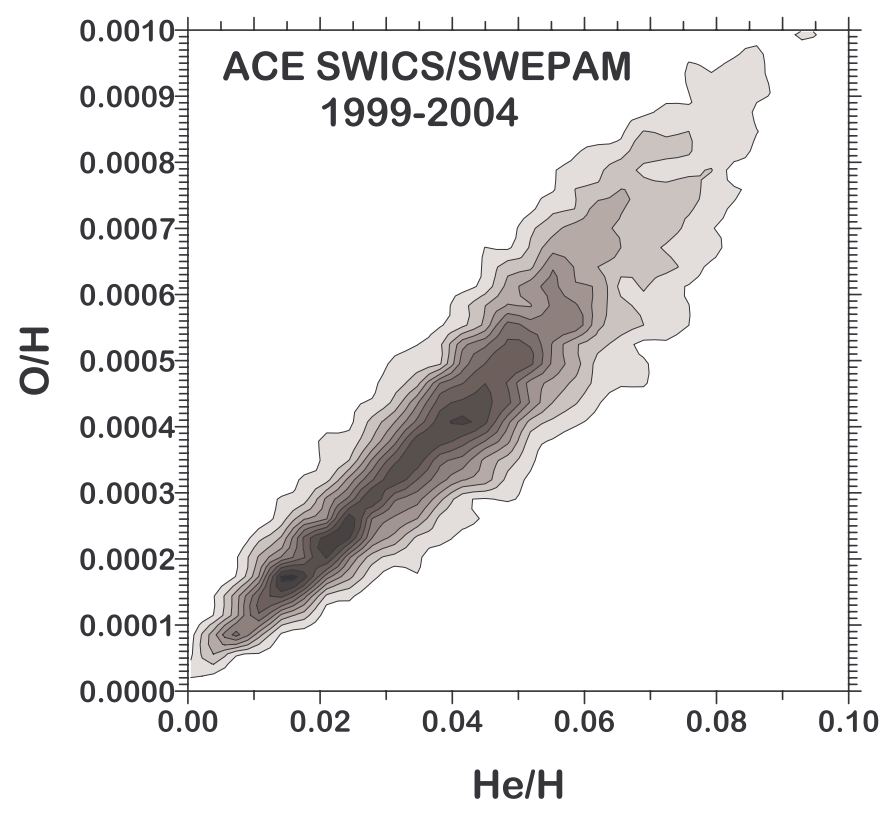

Fig. 3. The oxygen/hydrogen ratio in the solar wind varies over an order of magnitude. Its variation is strongly coupled with the helium/hydrogen ratio. We attribute the synchronicity of these variations to a common origin, which is unlikely a result of FIP fractionation, since ionization of oxygen and hydrogen are strongly coupled through resonant charge exchange. Both elements have very similar first ionization potentials and first ionization times (figure adapted from Bochsler et al. 2006).

$H_{i}$ given in Table 1 , we find $\frac{f_{4_{\mathrm{He}}}}{f_{20_{\mathrm{Ne}}}}=0.61 \pm 0.14$. Taking the flux-weighted average from the Apollo foil measurements of ${ }^{4} \mathrm{He} /{ }^{20} \mathrm{Ne}=570 \pm 70$, this yields for the elemental ratio in the OCZ:

$(\mathrm{He} / \mathrm{Ne})_{\mathrm{OCZ}}=870 \pm 120$,

corresponding to $[\mathrm{Ne}]_{\mathrm{OCZ}}=7.98 \pm 0.06$.

The neon/oxygen abundance ratio in the solar wind has been determined with several space experiments. The ISEE3/ICI-experiment used a combination of Wien filter with an energy per charge analyser. Bochsler et al. (1986) determined $[\mathrm{Ne} / \mathrm{O}]=0.17 \pm 0.02$ with this instrument. Their value is possibly somewhat biased towards abundances in low-speed solar wind. Collier et al. (1996) obtained similar values with WIND/MASS for in-ecliptic solar wind for a period of relatively low solar activity from December 1994 through August 1995. They find $[\mathrm{Ne} / \mathrm{O}]=0.14 \pm 0.01$ and no significant dependence of this ratio from the solar wind speed. It is worth noting, that also the coronal abundance ratio derived from solar energetic particles, which in this case is unlikely to suffer drastic mass/charge fractionation due to the relatively small differences in mass and charge, comes very close to this value with $[\mathrm{Ne} / \mathrm{O}]=0.153 \pm 0.004$ (Reames 1995).

Relating the oxygen abundance of the solar wind to the helium abundance gives a consistent picture: Bochsler et al. (1986) find $[\mathrm{He} / \mathrm{O}]=75 \pm 20$, whereas Collier et al. (1996) find $[\mathrm{He} / \mathrm{O}]=70 \pm 6$. The averages of Collier et al. (1996) are shown in Fig. 4. The dashed line in Fig. 4 is a least-square one-parameter fit to the data with the Coulomb drag model of Bodmer \& Bochsler (1998). Note again that shape and slope of this curve are unambiguously determined by the Coulomb-drag factors given in Table 1 and not derived from the fit to the data.

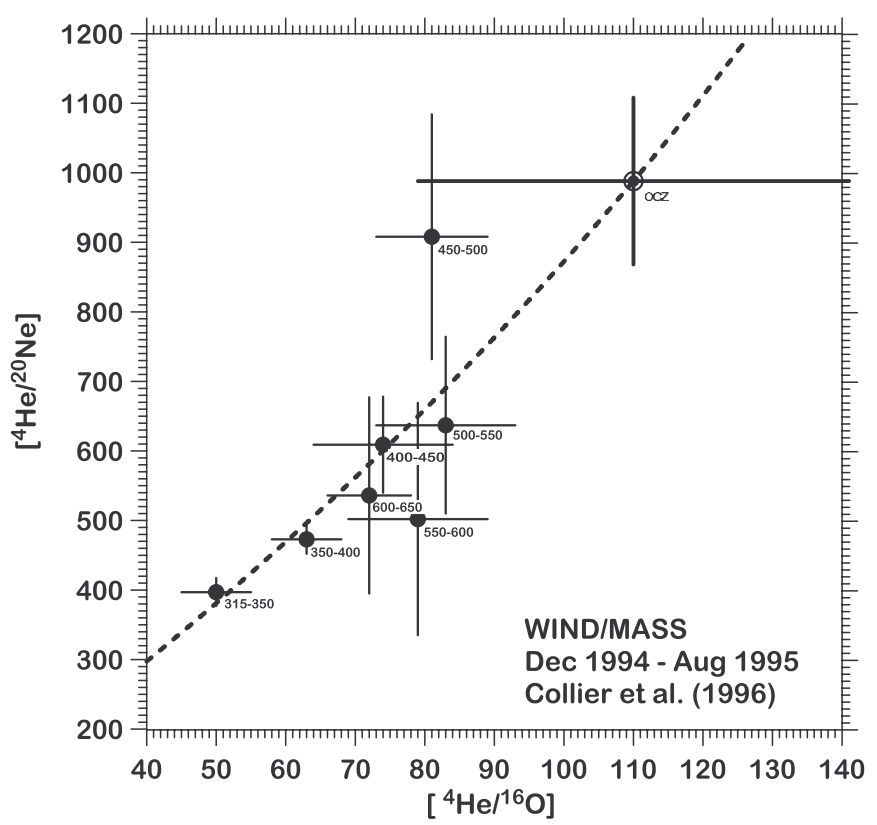

Fig. 4. He/Ne vs. He/O abundance ratios from Collier et al. (1996). The dashed line is a fit with the Coulomb drag model of Bodmer \& Bochsler (1998). Labels indicate the velocity bin in $\mathrm{km} / \mathrm{s}$ corresponding to the data points. The ordering of the helium abundances according to velocities corresponds to expectations for a Coulomb drag model, and long-range averages of $\mathrm{He} / \mathrm{H}$ abundance ratios observed by Aellig et al. (2001) and by Kasper et al. (2007). The large scatter of data points in the higher velocity bins could possibly be caused by an admixture of CME-ejecta. The solar point is a model extrapolation for the $\mathrm{OCZ}$ of the Sun (cf. Bodmer \& Bochsler 1998).

Applying the Coulomb-drag factors given in Table 1 and a typical $\mathrm{He} / \mathrm{H}$ fractionation factor for the period of WIND measurements of $f_{4}=0.50 \pm 0.15$ (Aellig et al. 2001) yields $\frac{f_{4} \mathrm{He}}{f_{16_{\mathrm{O}}}}=0.68 \pm 0.13$ and $\frac{f_{\mathrm{Ne}}}{f_{\mathrm{O}}}=1.11 \pm 0.04$. Consequently, the elemental abundance ratios derived from the observations of Collier et al. (1996) for the OCZ amount to

$(\mathrm{He} / \mathrm{O})_{\mathrm{OCZ}}=110 \pm 31$,
$(\mathrm{He} / \mathrm{Ne})_{\mathrm{OCZ}}=913 \pm 110$,
$(\mathrm{Ne} / \mathrm{O})_{\mathrm{OCZ}}=0.13 \pm 0.04$

The relatively large error bars are again due to the large uncertainties of the $\mathrm{He} / \mathrm{H}$ abundance at the time of the observations. They could possibly be reduced with a correlated data analysis.

\section{Conclusions}

Table 2 summarizes our results for the $\mathrm{He}, \mathrm{Ne}$, and $\mathrm{O}$ abundances of the OCZ of the Sun.

Our error bars include the $30 \%$ uncertainty originating from the estimate of the helium abundance and the experimental uncertainties of the abundance measurements of the solar wind. However, no allowance is left for systematic errors inherent in the model. Evidently, our solar wind derived oxygen and neon abundances are compatible with the values given by Grevesse \& Sauval (1998), but not with the ones given by Asplund et al. (2005). Our solar neon/oxygen abundance ratio is within error bars compatible with the recently derived photospheric value from EUV-spectra from SOHO/CDS (Young 2005). Our solar neon abundance is marginally compatible with Asplund et al. (2005) and Grevesse \& Sauval (1998), but clearly not with the 
Table 2. Abundances in the Outer Convective Zone of the Sun (in logarithmic dex units with $[\mathrm{H}] \equiv 12.00$ ).

\begin{tabular}{lcccc}
\hline \hline Element & $\begin{array}{c}\text { Grevesse \& } \\
\text { Sauval (1998) }\end{array}$ & $\begin{array}{c}\text { Asplund et al. } \\
(2005)\end{array}$ & $\begin{array}{c}\text { Bahcall et al. } \\
(2005)\end{array}$ & This work \\
\hline $\mathrm{H}$ & & \multicolumn{2}{c}{$\equiv 12.00$} & \\
\hline $\mathrm{He}$ & {$[10.93 \pm 0.004]^{a}$} & {$[10.93 \pm 0.01]$} & & $\equiv 10.914 \pm 0.003^{b}$ \\
$\mathrm{O}$ & $8.83 \pm 0.06$ & $8.66 \pm 0.05$ & - & $8.87 \pm 0.11$ \\
$\mathrm{Ne}$ & {$[8.08 \pm 0.06]$} & {$[7.84 \pm 0.06]$} & $8.29 \pm 0.05$ & $7.96 \pm 0.13$ \\
\hline
\end{tabular}

${ }^{a}$ Values in brackets: No direct photospheric measurement exists.

${ }^{b}$ Adopted from Gough (2006).

value proposed by Bahcall et al. (2005). Although photospheric abundances derived from optical spectra from three-dimensional models generally deserve higher credibility than abundances derived from one-dimensional photospheric models, a critical investigation of hidden model-dependent systematic errors seems justified. Furthermore, we believe that a comparison of photospheric elemental abundance ratios from different sources, which might exclude some hidden model uncertainties, rather than comparing absolute elemental abundances, could help to resolve the ongoing controversy.

From observations under different solar wind regimes, it appears that the Coulomb drag fractionation model of Bodmer \& Bochsler (1998) is applicable for not only explaining the variability of isotopic ratios in the solar wind but also for explaining the variability among high-FIP elements and for inferring the abundances of those elements in the OCZ of the Sun. In return: if our hypothesis on Coulomb drag being the main agent driving the variability of high-FIP elemental ratios in the solar wind is correct, it is certainly also applicable for isotopic ratios. At this time, the most important application will be the derivation of the isotopic composition of the OCZ from Genesis observations. Bodmer \& Bochsler (1998) and Wiens et al. (2004) have used the model to infer the isotopic composition of helium and neon from the Apollo foil measurements. Their extrapolation rested on inferences on the ${ }^{4} \mathrm{He} /{ }^{3} \mathrm{He}$ ratio in high-speed solar wind measured with Ulysses/SWICS. We have now modified this baseline and assumed that the $\mathrm{He} / \mathrm{H}$ abundance ratio during the exposure of the Apollo foils was in the range of $0.040 \pm 0.012$. With this modification we find that

$\left({ }^{4} \mathrm{He} /{ }^{3} \mathrm{He}\right)_{\mathrm{OCZ}}=3240 \pm 560$,

and

$\left({ }^{20} \mathrm{Ne} /{ }^{22} \mathrm{Ne}\right)_{\mathrm{OCZ}}=13.3 \pm 0.3$.
Acknowledgements. The author has taken profit from helpful discussions with Douglas Gough, Ansgar Grimberg, and Nicolas Grevesse, and he gratefully acknowledges support by the Swiss National Science Foundation.

\section{References}

Aellig, M. R., Lazarus, A. J., \& Steinberg, J. T. 2001, Geophys. Res. Lett., 28, 2767

Antia, H. M., \& Basu, S. 2006, ApJ, 644, 1292

Asplund, M., Grevesse, N., \& Sauval, A. J. 2005, in Cosmic Abundances as Records of Stellar Evolution and Nucleosynthesis, ed. T. G. Barnes, \& F. N. Bash, ASP Conf. Ser., 336, 25

Bahcall, J. N., Basu, S., \& Serenelli, A. M. 2005, ApJ, 631, 1281

Bochsler, P., Auchère, F., \& Skoug, R. M. 2006, in Proc. SOHO 17 Conf., Taormina, Italy (ESA SP 617)

Bochsler, P., Geiss, J., \& Kunz, S. 1986, Sol. Phys., 103, 177

Bodmer, R., \& Bochsler, P. 1998, A\&A, 337, 921

Collier, M. R., Hamilton, D. C., Gloeckler, G., Bochsler, P., \& Sheldon, R. B. 1996, Geophys. Res. Lett., 23, 1191

Feldman, W. C., Asbridge, J. R., Bame, S. J., \& Gosling, J. T. 1978, J. Geophys. Res., 83, 2177

Geiss, J., \& Bochsler, P. 1985, in Rapports isotopiques dans le système solaire (Cépaduès Editions), 213

Geiss, J., Bühler, F., Cerutti, H., Eberhardt, P., \& Filleux, C. 1972, Apollo 16 Prel. Sci. Rep. NASA Special Publication, 315, 14.1

Geiss, J., Bühler, F., Cerutti, H., et al. 2004, Space Sci. Rev., 110, 307

Gough, D. O. 2006, in Proc. SOHO 17 Conference, Taormina, Italy (ESA SP 617)

Grevesse, N., \& Sauval, A. J. 1998, Space Sci. Rev., 85, 161

Grimberg, A., Baur, H., Burnett, D. S., Bochsler, P., \& Wieler, R. 2007, Lunar Planetary Science Conference, XXXVIII, \#1270

Kasper, J. C., Lazarus, A. J., Steinberg, J. T., Ogilvie, K. W., \& Szabo, A. 2006, J. Geophys. Res., 111

Kasper, J. C., Stevens, M. L., Lazarus, A. J., Steinberg, J. T., \& Ogilvie, K. W. 2007, ApJ, 660, 901

Kunz, S. 1983, PhD Thesis, University of Bern, Switzerland

Reames, D. V. 1995, Adv. Space Res., 15, 41

Wiens, R. C., Bochsler, P., Burnett, D. S., \& Wimmer-Schweingruber, R. F. 2004, Earth Planet. Sci. Lett., 222, 697

Young, P. R. 2005, A\&A, 444, L45 